\title{
JD14
}

\section{The First Results of Hipparcos and Tycho}

\author{
Chairperson and Editor: C. Turon
}

